\title{
CORREALTION OF CT CHEST IMAGES WITH THE CLINICAL PRESENTATION IN COVID-19 INFECTION: A CLINICO-RADIOLOGICAL STUDY
}

KEY WORDS: COVID-19, CT Imaging, Pandemic

\section{Dr Uzma Khan \\ Dr Arindam Ghosh \\ DrVikram Mutneja*}

Ex Professor And Head, BJS Dental College, Ludhiana.

Consultant GI Surgeon, Saudi German Hospital, Dubai.

Consultant, Radiology Department, SPS Hospital, Ludhiana.*Corresponding Author

Aim: The study aimed to evaluate the correlation of CT chest images with the clinical presentation in COVID-19 infection.

Methodology: 50 confirmed COVID-19 patients those who were hospitalized were included in the study. Patient history, laboratory results, demographic data, severity, time course of symptoms was recorded. The duration of onset of the symptoms to initial CT scan, fever recordings including duration of onset of fever to CT scanning and white blood counts were recorded along with the CT Scores of each patient.

Results: The CT scores were found to have significant correlation with age $(r=0.348, p$ value $=0.046)$, duration of onset to CT scanning $(r=0.654$, $p$ value $=0.001)$, duration of fever onset to CT scanning $(r=0.679, p$ value $=0.001)$ and white blood cell count $(r=0.398$, $p$ value $=0.034)$.

Conclusion: We found correlation between the CT scores and Age, duration of onset to Ct scanning, duration of fever onset to Ct scanning and white blood cell count.

\section{INTRODUCTION}

In December 2019, a new infectious disease emerged in Wuhan, China named COVID-19 which soon became pandemic and spread worldwide. The cause of the disease was a novel betacoronavirus, the severe acute respiratory syndrome coronavirus 2 (SARS-CoV-2). ${ }^{1}$ COVID-19 had impacted the whole world adversely. It is essential to understand the epidemiological features, clinical presentation, recovery and mortality of COVID-19 for implementing the management strategies of the disease.

The current understanding of the disease suggest that the incubation period of the infection is usually $3-7$ days to a maximum of 14 days. $^{2}$ The impact of disease in elderly patients has found to be more pronounced especially those with associated underlying diseases. ${ }^{3}$ This infection can affect the individual of any age including children and infants. The patients admitting in hospitals are majorly presented with co morbidities including diabetes, hypertension, cardiovascular and cerebrovascular diseases.

The clinical presentation of COVIDl-19 in the prodromal phase includes the fever, dry cough, headache, malaise, loss of smell and loss of taste which is definitely not specific. ${ }^{3}$ Chest computed tomography (CT) can be referred as a valuable tool to identify the patient with COVID-19 infections at an initial stage during which the symptoms are not specified. ${ }^{6,7}$ Chest CT can be considered an indispensible component for confirmed diagnosis in suspected patients. Both the clinical and the radiological manifestations are important for the definitive diagnosis and to evaluate the progression of the infection.

Till date the correlation between the clinical presentation and the CT images are unclear. Therefore, the present study was aimed to analyze the initial CT images of the patients suffering from COVID-19 to understand the lung abnormalities and their association with the clinical features.

\section{METHODOLOGY}

The data for the present study was taken from the records of 50 confirmed COVID-19 patients those who were hospitalized. Patient history, laboratory results, demographic data, severity, time course of symptoms was recorded. The duration of onset of the symptoms to initial CT scan, fever recordings including duration of onset of fever to CT scanning and white blood counts were recorded.
CT scans were assessed by two highly experienced radiologists that have used the scoring criteria established by Chung et al. (2020) ${ }^{9}$ to examine the intensity of involvement of each of the five lung lobes. The scoring is explained as follows:

Score 0 -normality;

Score $1-1 \%-25 \%$ of the total lobe area involved;

Score $2-26 \%-50 \%$ of the total lobe area involved;

Score $3-51 \%-75 \%$ of the total lobe area involved;

Score $4-76 \%-100 \%$ of the total lobe area involved.

The overall lung "CT score" was calculated by adding the five lung lobe scores (range-0-20).

\section{STATISTICAL ANALYSIS}

Data were tabulated and examined using the Statistical Package for Social Sciences Version 20.0 (IBM SPSS Statistics for Mac, Armonk, NY: IBM Corp, USA). Descriptive statistical analysis had been carried out in the present study. Results on categorical measurements are presented as frequency distribution. The statistical power calculation was based on the assumption that the data were normally distributed. The data were subjected to Pearson correlation test to identify the correlation between the initial CT scans and clinical features. A P $<0.05$ was considered as significant.

\section{RESULTS}

The patients enrolled in the present study had a median age of 43 years (Range $=33-56$ ). 29 females and 21 males were enrolled in the study. The median duration between onset and initial CT was 5 days which ranges between $3-7$ days among the patients. Out of the study population 39 patients presented fever as their initial symptoms and 11 patients had temperature below 37.3 degrees. 36 patients out of 39 patients had mild to moderate fever below 39 degrees. The median duration between onset of fever and CT scanning was 4 days ranging from $1-6$ days. On hospitalization, 18 patients were presented with leucopenia. (Table l)

Table 2 presents the correlation of the CT score and the clinical data. CT scores were found to be positively correlated with the age $(r=0.348$, $p$ value $=0.046)$. The sex of the patient is not found to be correlated with the CT scores $(r=0.193, p$ value $=0.09$ ). The correlation between duration of onset to CT scanning was found to be positively correlated ( $r=0.654, p$ value $=0.001$ ) along with the positive correlation with the duration of fever onset to CT scanning $(r=0.679, p$ value $=$ 
$0.001)$. No significant correlation was found between the highest temperature and the CT scores $(r=0.280, p$ value $=$ 0.08). CT scores were also positively correlated with the white blood cell count $(r=0.398$, $p$ value $=0.034)$.

\section{DISCUSSION}

The present study was conducted with the vision of finding the correlation between the CT scores and the clinical features. In the present study the lung involvement was found to be correlated with the duration of the clinical symptoms. The lung abnormalities were confirmed with the initial CT images of each patient. Bernheim et al. ${ }^{10}$ have presented that the lung involvement rapidly increases with the onset of symptoms.

Our study presented that $78 \%$ patients showed the symptom of fever and similar reports were presented by the studies conducted byWang et al. ${ }^{11}$

$\mathrm{Wu}$ et al. ${ }^{12}$ have also stated that the lung lesion positively correlated with the degree of fever but the present study did not find a significant correlation between the highest temperature and the CT scores.

The pathophysiology of the COVID-19 is vaguely understood by the researchers. The preset understanding states that the SARS -CoV-2 infection initiates the increase in proinflammatory cytokines like interleukin- $1 \beta$ (IL-1 $\beta$ ), IL-4, IL-10, interferon- $\gamma$ (IFN- $\gamma$ ), IFN- $\gamma$ - inducible protein-10 (IP10), and monocyte chemoattractant protein-l (MCP1)). ${ }^{5}$ Over activation of the T cells leads to spread of the virus particle through the respiratory mucosa and infect other cells which in turn induce the cytokine storm in the body. The storm of the cytokines generate a series of events at molecular level causing changes in the white blood cells and immune cells resulting in alveolar damage. ${ }^{12}$

The present study had found positive correlation between the white blood cell count and the CT scores. The present data is supported by the study conducted by Wu et al. ${ }^{13}$ stating the correlation between the degree of lung lesion and the white blood cell count.

Further studies are required to evaluate the missing pieces of the pathophysiology of the COVID-19 infection which will help in finding the correct management strategies which is lacking till date.

The present study has certain limitations, firstly the sample size of the study was small and majority of patients has mild symptoms. Therefore, it is necessary to further conduct the study in association with the patients with severe illness following COVID-19 exposure. Secondly, the limited data was available due to outbreak situation in hospitals which made it impossible to correlate certain other clinical data with the CT scoring. Thirdly, the continued course of the disease in association with the CT scoring was lacking. Therefore, it is essential to validate the results of the present study with further prospective studies to evaluate the confirmatory association of CT images and the progression of the clinical symptoms.

\section{CONCLUSION}

Within the limitation of the study we found correlation between the CT scores and Age, duration of onset to $\mathrm{Ct}$ scanning, duration of fever onset to $\mathrm{Ct}$ scanning and white blood cell count. It is necessary to evaluate the correct understanding of the pathophysiology of the COVID-19 infection to formulate a correct management strategy. To correlate the disease severity and the CT images further prospective studies are needed to validate the present outcomes.

Table 1: Representation of the demographic data and the clinical features of the patients $(N=50)$

\begin{tabular}{|l|l|}
\hline Parameters & Value \\
\hline www.worldwidejournals.com
\end{tabular}

\begin{tabular}{|l|l|}
\hline Sex & \\
Male & $21(42)$ \\
Female & $29(58)$ \\
\hline Age & $43(33-56)$ \\
\hline Exposure history & \\
Recent travel history & $24(48)$ \\
Exposure from infected & \\
patient & $16(32)$ \\
Unknown exposure & $10(20)$ \\
\hline Fever & Highest temperature- 38.6 \\
& $($ range 37.4-38.8) \\
$<37.3$ & $11(22)$ \\
$37.3-38.0$ & $17(34)$ \\
38.1-39.0 & $19(38)$ \\
$>39.0$ & $3(6)$ \\
\hline Other symptoms & $35(70)$ \\
Cough & $23(46)$ \\
Sputum production & $22(44)$ \\
Sore throat & $20(40)$ \\
Chills/rigor & $18(36)$ \\
Rhinorrhoea & $27(54)$ \\
Shortness of breath & $11(22)$ \\
Headache/dizziness & $22(44)$ \\
Muscle pain/fatigue & $6(12)$ \\
Diarrhea & $5(3-7)$ \\
\hline Duration from onset to CT \\
scanning (d)
\end{tabular}

Table 2: Representation of the correlation between the clinical features and the CT scores

\begin{tabular}{|l|l|l|}
\hline Parameters & $\boldsymbol{r}$ value & P value \\
\hline Age & 0.348 & $0.046^{*}$ \\
\hline sex & 0.193 & 0.09 \\
\hline $\begin{array}{l}\text { Duration from onset to CT } \\
\text { scanning }\end{array}$ & 0.654 & $0.001^{*}$ \\
\hline $\begin{array}{l}\text { Duration from fever onset to CT } \\
\text { scanning }\end{array}$ & 0.679 & $0.001^{*}$ \\
\hline Highest Temperature & 0.280 & 0.083 \\
\hline White blood cell count & 0.398 & $0.034^{*}$ \\
\hline
\end{tabular}

* $p$ value is significant ( $p$ value $<0.05$ )

\section{REFERENCES}

1. Lu RJ, Zhao X, Li J, et al., 2020. Genomic characterisation and epidemiology of 2019 novel coronavirus: implications for virus origins and receptor binding. Lancet, 395(10224):565-574.

2. Sun K, Chen J, Viboud C. Early epidemiological analysis of the coronavirus disease 2019 outbreak based on crowd sourced data: a population-level observational study. The Lancet Digital Health. 2020

3. Yang X, Yu Y, Xu J, et al. Clinical course and outcomes of critically ill patients with SARS-CoV-2 pneumonia in Wuhan, China: a single-centered, retrospective, observational study.Lancet Respir Med. 2020.

4. Chen N, Zhou M, Dong X, et al. Epidemiological and clinical characteristics of 99 cases of 2019 novel coronavirus pneumonia in Wuhan, China: a descriptive study.Lancet. 2020

5. Huang C, Wang Y, Li X, et al. Clinical features of patients with 2019 novel coronavirus in Wuhan, China. Lancet. 2020; 183-5

6. Fang Y, Zhang H, Xie J, et al. Sensitivity of chest CT for COVID-19: comparison to RT-PCR. Radiology. 2020;200432.

7. Xie X, Zhong Z, Zhao W, et al. Chest CT for typical 2019-nCoV pneumonia: relationship to negative RT-PCR testing. Radiology.2020;200343.

8. WHO. Preliminary clinical description of severe acute respiratory syndrome. Available at: https://www.who.int/csr/sars/clinical/en/.Accessed March 21, 2003.

9. Chung M, Bernheim A, Mei XY, et al., 2020. CT imaging features of 2019 novel coronavirus (2019-nCoV). Radiology,295(1):200230.

10. Bernheim A, Mei XY, Huang MQ, et al., 2020. Chest CT findings in coronavirus disease-19 (COVID-19): relationship to duration of infection. Radiology, online.

11. Wang DW, Hu B, Hu C, et al., 2020. Clinical characteristics of 138 hospitalized patients with 2019 novel coronavirus infected pneumonia in Wuhan, China. JAMA, 323(11): 1061-1069.

12. Xu Z, Shi L,Wang YJ, et al., 2020. Pathological findings of COVID-19 associated with acute respiratory distress syndrome. Lancet Respir Med,8(4):420-422. 
PARIPEX - INDIAN JOURNAL OF RESEARCH | Volume - 9 | Issue - 12 |December - 2020 | PRINT ISSN No. 2250 - 1991 | DOI : $10.36106 /$ paripex

13. Wu J, Wu XJ, Zeng WB, et al., 2020. Chest CT findings in patients with coronavirus disease 2019 and its relationship with clinical features. Invest Radiol,55(5):257-261. 